\title{
NEW PROPERTIES OF THE INTERSECTION NUMBERS ON MODULI SPACES OF CURVES
}

\author{
Kefeng LiU AND HaO XU
}

\begin{abstract}
We present certain new properties about the intersection numbers on moduli spaces of curves $\overline{\mathcal{M}}_{g, n}$, including a simple explicit formula of $n$-point functions and several new identities of intersection numbers. In particular we prove a new identity, which together with a conjectural identity implies the famous Faber's conjecture about relations in $\mathcal{R}^{g-2}\left(\mathcal{M}_{g}\right)$. These new identities clarify the mysterious constant in Faber's conjecture and uncover novel combinatorial structures of intersection numbers.

We also discuss some numerical properties of Hodge integrals which have provided numerous inspirations for this work.
\end{abstract}

\section{Introduction}

Denote by $\overline{\mathcal{M}}_{g, n}$ the moduli space of stable $n$-pointed genus $g$ complex algebraic curves. We have the forgetting the last marked point morphism

$$
\pi: \overline{\mathcal{M}}_{g, n+1} \longrightarrow \overline{\mathcal{M}}_{g, n} \text {. }
$$

Denote by $\sigma_{1}, \ldots, \sigma_{n}$ the canonical sections of $\pi$, and by $D_{1}, \ldots, D_{n}$ the corresponding divisors in $\overline{\mathcal{M}}_{g, n+1}$. Let $\omega_{\pi}$ be the relative dualizing sheaf, we have the following tautological classes on moduli spaces of curves.

$$
\begin{aligned}
\psi_{i} & =c_{1}\left(\sigma_{i}^{*}\left(\omega_{\pi}\right)\right) \\
\kappa_{i} & =\pi_{*}\left(c_{1}\left(\omega_{\pi}\left(\sum D_{i}\right)\right)^{i+1}\right) \\
\lambda_{l} & =c_{l}\left(\pi_{*}\left(\omega_{\pi}\right)\right), \quad 1 \leq l \leq g .
\end{aligned}
$$

The classes $\kappa_{i}$ were first introduced by Mumford [21] on $\overline{\mathcal{M}}_{g}$, their generalization to $\overline{\mathcal{M}}_{g, n}$ here is due to Arbarello and Cornalba [1].

We use Witten's notation in this paper,

$$
\left\langle\tau_{d_{1}} \cdots \tau_{d_{n}} \lambda_{b_{1}} \cdots \lambda_{b_{k}}\right\rangle_{g}:=\int_{\overline{\mathcal{M}}_{g, n}} \psi_{1}^{d_{1}} \cdots \psi_{n}^{d_{n}} \lambda_{b_{1}} \cdots \lambda_{b_{k}} .
$$

The moduli space of curves is a central object of study in algebraic geometry. The intersection theory of tautological classes on the moduli space of curves is a very important subject and has close connections to string theory, quantum gravity and many branches of mathematics.

Intersection numbers involving only $\psi$ classes can be computed recursively by the celebrated Witten's conjecture [24] (proved by Kontsevich [17]) or by the formula of $n$-point functions [19]. General intersections involving $\psi, \lambda, \kappa$ or boundary divisor classes can be reduced to intersections of $\psi$ classes by Faber's algorithm [7].

Received by the editors November 9, 2006. 
The following form of Witten's conjecture is called the DVV formula [4].

$$
\begin{aligned}
\left\langle\tau_{k+1} \tau_{d_{1}} \cdots \tau_{d_{n}}\right\rangle_{g} & =\frac{1}{(2 k+3) ! !}\left[\sum_{j=1}^{n} \frac{\left(2 k+2 d_{j}+1\right) ! !}{\left(2 d_{j}-1\right) ! !}\left\langle\tau_{d_{1}} \cdots \tau_{d_{j}+k} \cdots \tau_{d_{n}}\right\rangle_{g}\right. \\
+ & \frac{1}{2} \sum_{r+s=k-1}(2 r+1) ! !(2 s+1) ! !\left\langle\tau_{r} \tau_{s} \tau_{d_{1}} \cdots \tau_{d_{n}}\right\rangle_{g-1} \\
+ & \left.\frac{1}{2} \sum_{r+s=k-1}(2 r+1) ! !(2 s+1) ! ! \sum_{\underline{n}=I \amalg J}\left\langle\tau_{r} \prod_{i \in I} \tau_{d_{i}}\right\rangle_{g^{\prime}}\left\langle\tau_{s} \prod_{i \in J} \tau_{d_{i}}\right\rangle_{g-g^{\prime}}\right]
\end{aligned}
$$

where $\underline{n}=\{1,2, \ldots, n\}$.

In 1993, Carel Faber [6] proposed his remarkable conjectures about the structure of tautological ring $\mathcal{R}^{*}\left(\mathcal{M}_{g}\right)$. In the past decade, Faber's conjecture motivated a tremendous progress toward understanding of the topology of moduli spaces of curves. For background materials, we recommend Vakil's excellent survey [23].

An important part of Faber's conjectures is the famous Faber's intersection number conjecture, which is the following relations in $\mathcal{R}^{g-2}\left(\mathcal{M}_{g}\right)$, if $\sum_{j=1}^{n} d_{j}=g-2$,

$$
\sum_{\sigma \in S_{n}} \kappa_{\sigma}=\frac{(2 g-3+n) !(2 g-1) ! !}{(2 g-1) ! \prod_{j=1}^{n}\left(2 d_{j}+1\right) ! !} \kappa_{g-2},
$$

where $\sum_{\sigma \in S_{n}} \kappa_{\sigma}=\left(\pi_{1} \ldots \pi_{n}\right)_{*}\left(\psi_{1}^{d_{1}+1} \ldots \psi_{n}^{d_{n}+1}\right)$ and $\kappa_{\sigma}$ is defined as follows. Write the permutation $\sigma$ as a product of $\nu(\sigma)$ disjoint cycles, including 1-cycles: $\sigma=$ $\beta_{1} \cdots \beta_{\nu(\sigma)}$, where we think of the symmetric group $S_{n}$ as acting on the $n$-tuple $\left(d_{1}, \ldots, d_{n}\right)$. Denote by $|\beta|$ the sum of the elements of a cycle $\beta$. Then $\kappa_{\sigma}=$ $\kappa_{\left|\beta_{1}\right|} \kappa_{\left|\beta_{2}\right|} \ldots \kappa_{\left|\beta_{\nu(\sigma)}\right|}$.

By the work of Looijenga [20] and Faber [5], we know that $\mathcal{R}^{g-2}\left(\mathcal{M}_{g}\right)=\mathbb{Q}$ is 1-dimensional and $\lambda_{g} \lambda_{g-1}$ vanishes on the boundary of $\overline{\mathcal{M}}_{g}$. So Faber's intersection number conjecture is equivalent to the following Hodge integral identity.

If $d_{j} \geq 1$ and $\sum_{j=1}^{n}\left(d_{j}-1\right)=g-2$,

$$
\begin{aligned}
\int_{\overline{\mathcal{M}}_{g, n}} \psi_{1}^{d_{1}} \ldots \psi_{n}^{d_{n}} \lambda_{g} \lambda_{g-1} & =\int_{\overline{\mathcal{M}}_{g}} \sum_{\sigma \in S_{n}} \kappa_{\sigma} \lambda_{g} \lambda_{g-1} \\
& =\frac{(2 g-3+n) !(2 g-1) ! !}{(2 g-1) ! \prod_{j=1}^{n}\left(2 d_{j}-1\right) ! !} \int_{\overline{\mathcal{M}}_{g}} \kappa_{g-2} \lambda_{g} \lambda_{g-1} \\
& =\frac{(2 g-3+n) !\left|B_{2 g}\right|}{2^{2 g-1}(2 g) ! \prod_{j=1}^{n}\left(2 d_{j}-1\right) ! !} .
\end{aligned}
$$

where $\int_{\overline{\mathcal{M}}_{g}} \kappa_{g-2} \lambda_{g} \lambda_{g-1}=\frac{\left|B_{2 g}\right|(g-1) !}{2^{g}(2 g) !}$ is proved by Faber [5].

Since $\lambda_{g} \lambda_{g-1}=(-1)^{g-1}(2 g-1) ! \cdot \operatorname{ch}_{2 g-1}(\mathbb{E})$, we use Mumford's formula [21] for the Chern character of Hodge bundles

$$
\operatorname{ch}_{2 g-1}(\mathbb{E})=\frac{B_{2 g}}{(2 g) !}\left[\kappa_{2 g-1}-\sum_{i=1}^{n} \psi_{i}^{2 g-1}+\frac{1}{2} \sum_{\xi \in \Delta} l_{\xi_{*}}\left(\sum_{i=0}^{2 g-2} \psi_{n+1}^{i}\left(-\psi_{n+2}\right)^{2 g-2-i}\right)\right]
$$


to get

$$
\begin{aligned}
& \frac{(2 g-3+n) !}{2^{2 g-1}(2 g-1) !} \cdot \frac{1}{\prod_{j=1}^{n}\left(2 d_{j}-1\right) ! !} \\
& =\left\langle\tau_{d_{1}} \cdots \tau_{d_{n}} \tau_{2 g}\right\rangle_{g}-\sum_{j=1}^{n}\left\langle\tau_{d_{1}} \cdots \tau_{d_{j-1}} \tau_{d_{j}+2 g-1} \tau_{d_{j+1}} \cdots \tau_{d_{n}}\right\rangle_{g} \\
& +\frac{1}{2} \sum_{j=0}^{2 g-2}(-1)^{j}\left\langle\tau_{2 g-2-j} \tau_{j} \tau_{d_{1}} \cdots \tau_{d_{n}}\right\rangle_{g-1} \\
& \quad+\frac{1}{2} \sum_{\underline{n}=I} \amalg \sum_{j=0}^{2 g-2}(-1)^{j}\left\langle\tau_{j} \prod_{i \in I} \tau_{d_{i}}\right\rangle_{g^{\prime}}\left\langle\tau_{2 g-2-j} \prod_{i \in J} \tau_{d_{i}}\right\rangle_{g-g^{\prime}}
\end{aligned}
$$

where $d_{j} \geq 1, \sum_{j=1}^{n}\left(d_{j}-1\right)=g-2$ and $\underline{n}=\{1,2, \ldots, n\}$.

In fact, the proportional constant in identity (1) is observed experimentally by Faber from identity (3), through implementing Witten's conjecture to calculate intersection numbers.

In this paper, we will abuse terminology by calling either of the equivalent identities (1), (2) and (3) the Faber's conjecture.

Getzler and Pandharipande [11] derive Faber's conjecture from the degree $0 \mathrm{Vi}$ rasoro conjecture for $\mathbb{P}^{2}$. On the other hand, Givental [13] has announced a proof of Virasoro conjecture for $\mathbb{P}^{n}$. Y.P. Lee and R. Pandharipande are writing a book supplying the details. Recently, Goulden, Jackson and Vakil [14] have given a more direct and enlightening proof of Faber's conjecture for up to three points and explained their approach for the general case. Their method of proof is a marvelous synthesis of geometry and combinatorics, which has already found other elegant applications $[3,15]$.

In this paper, we present a series of simple new identities of intersection numbers, aiming to clarify combinatorial structures in Faber's conjecture.

The explicit formula of $n$-point functions (as stated in theorem 2.8 and proved in [19]) for intersection numbers will play an important role in this work.

\section{Acknowledgements}

The authors would like to express our special thanks to Ravi Vakil for many helpful comments and discussions. We wish to thank Professor Enrico Arbarello, Sergei Lando and Edward Witten for useful comments and their interests in this work. We thank Professor Carel Faber for his wonderful Maple program for computing Hodge integrals and for communicating Zagier's three-point function to us. We also thank the anonymous referee for detailed comments that have greatly improved the manuscript.

\section{Several new identities of intersection numbers}

Now we announce the following identity of intersection numbers which clarifies the mysterious constants in Faber's conjecture. 
Theorem 2.1. Let $d_{j} \geq 1$ and $\sum_{j=1}^{n}\left(d_{j}-1\right)=g-1$. Then

$$
\sum_{j=0}^{2 g}(-1)^{j}\left\langle\tau_{2 g-j} \tau_{j} \tau_{d_{1}} \cdots \tau_{d_{n}}\right\rangle_{g}=\frac{(2 g-1+n) !}{2^{2 g}(2 g+1) !} \cdot \frac{1}{\prod_{j=1}^{n}\left(2 d_{j}-1\right) ! !}
$$

When $n=1$, identity (4) becomes

$$
\sum_{j=0}^{2 g}(-1)^{j}\left\langle\tau_{2 g-j} \tau_{j} \tau_{g}\right\rangle_{g}=\frac{1}{2^{2 g}(2 g+1) ! !}
$$

which has been proved in $[5,9]$.

From theorem 2.1, we see that Faber's conjecture (3) is equivalent to the following simpler identity.

Conjecture 2.2. Let $d_{j} \geq 0, \sum_{j=1}^{n} d_{j}=g+n-2$ and $\underline{n}=\{1,2, \ldots, n\}$. Then

$$
\begin{aligned}
\left\langle\tau_{d_{1}} \cdots \tau_{d_{n}} \tau_{2 g}\right\rangle_{g}= & \sum_{j=1}^{n}\left\langle\tau_{d_{1}} \cdots \tau_{d_{j-1}} \tau_{d_{j}+2 g-1} \tau_{d_{j+1}} \cdots \tau_{d_{n}}\right\rangle_{g} \\
& -\frac{1}{2} \sum_{\underline{n}=I \amalg J} \sum_{j=0}^{2 g-2}(-1)^{j}\left\langle\tau_{j} \prod_{i \in I} \tau_{d_{i}}\right\rangle_{g^{\prime}}\left\langle\tau_{2 g-2-j} \prod_{i \in J} \tau_{d_{i}}\right\rangle_{g-g^{\prime}}
\end{aligned}
$$

Accompanying identity (4) of theorem 2.1, we also have the following vanishing theorem of intersection numbers.

Theorem 2.3. Let $K>g, d_{j} \geq 0$ and $\sum_{j=1}^{n} d_{j}=3 g+n-2 K-1$. Then

$$
\sum_{j=0}^{2 K}(-1)^{j}\left\langle\tau_{2 K-j} \tau_{j} \tau_{d_{1}} \cdots \tau_{d_{n}}\right\rangle_{g}=0
$$

The following corollary of Theorem 2.3 is a complement to Conjecture 2.2.

Corollary 2.4. Let $K>g, d_{j} \geq 0$ and $\sum_{j=1}^{n} d_{j}=3 g+n-2 K-2$. Then

$$
\begin{aligned}
\left\langle\tau_{d_{1}} \cdots \tau_{d_{n}} \tau_{2 K}\right\rangle_{g}= & \sum_{j=1}^{n}\left\langle\tau_{d_{1}} \cdots \tau_{d_{j-1}} \tau_{d_{j}+2 K-1} \tau_{d_{j+1}} \cdots \tau_{d_{n}}\right\rangle_{g} \\
& -\frac{1}{2} \sum_{\underline{n}=I \amalg J} \sum_{j=0}^{2 K-2}(-1)^{j}\left\langle\tau_{j} \prod_{i \in I} \tau_{d_{i}}\right\rangle_{g^{\prime}}\left\langle\tau_{2 K-2-j} \prod_{i \in J} \tau_{d_{i}}\right\rangle_{g-g^{\prime}}
\end{aligned}
$$

Proof. The identity follows from Mumford's formula [21]

$$
\operatorname{ch}(\mathbb{E})=g+\sum_{k=1}^{\infty} \frac{B_{2 k}}{(2 k) !}\left[\kappa_{2 k-1}-\sum_{i=1}^{n} \psi_{i}^{2 k-1}+\frac{1}{2} \sum_{\xi \in \Delta} l_{\xi_{*}}\left(\sum_{i=0}^{2 k-2} \psi_{n+1}^{i}\left(-\psi_{n+2}\right)^{2 k-2-i}\right)\right]
$$

where $\operatorname{ch}_{2 k-1}(\mathbb{E})=0$, when $k>g$.

Amazingly we also found the following conjectural identity experimentally. Please compare with the identities (3) and (5), 
Conjecture 2.5. Let $g \geq 2, d_{j} \geq 1$ and $\sum_{j=1}^{n}\left(d_{j}-1\right)=g$. Then

$$
\begin{aligned}
& \frac{(2 g-3+n) !}{2^{2 g+1}(2 g-3) !} \cdot \frac{1}{\prod_{j=1}^{n}\left(2 d_{j}-1\right) ! !} \\
& =\left\langle\tau_{d_{1}} \cdots \tau_{d_{n}} \tau_{2 g-2}\right\rangle_{g}-\sum_{j=1}^{n}\left\langle\tau_{d_{1}} \cdots \tau_{d_{j-1}} \tau_{d_{j}+2 g-3} \tau_{d_{j+1}} \cdots \tau_{d_{n}}\right\rangle_{g} \\
& \quad+\frac{1}{2} \sum_{\underline{n}=I \amalg J} \sum_{j=0}^{2 g-4}(-1)^{j}\left\langle\tau_{j} \prod_{i \in I} \tau_{d_{i}}\right\rangle_{g^{\prime}}\left\langle\tau_{2 g-4-j} \prod_{i \in J} \tau_{d_{i}}\right\rangle_{g-g^{\prime}} .
\end{aligned}
$$

Since $(2 g-3) ! \cdot \mathrm{ch}_{2 g-3}(\mathbb{E})=(-1)^{g-1}\left(3 \lambda_{g-3} \lambda_{g}-\lambda_{g-1} \lambda_{g-2}\right)$, it's easy to see that the above identity (8) is equivalent to the following identity of Hodge integrals,

Conjecture 2.6. Let $g \geq 2, d_{j} \geq 1$ and $\sum_{j=1}^{n}\left(d_{j}-1\right)=g$. Then

$$
\begin{aligned}
& \frac{2 g-2}{\left|B_{2 g-2}\right|}\left(\int_{\overline{\mathcal{M}}_{g, n}} \psi_{1}^{d_{1}} \cdots \psi_{n}^{d_{n}} \lambda_{g-1} \lambda_{g-2}-3 \int_{\overline{\mathcal{M}}_{g, n}} \psi_{1}^{d_{1}} \cdots \psi_{n}^{d_{n}} \lambda_{g-3} \lambda_{g}\right) \\
& \quad=\frac{1}{2} \sum_{j=0}^{2 g-4}(-1)^{j}\left\langle\tau_{2 g-4-j} \tau_{j} \tau_{d_{1}} \cdots \tau_{d_{n}}\right\rangle_{g-1}+\frac{(2 g-3+n) !}{2^{2 g+1}(2 g-3) !} \cdot \frac{1}{\prod_{j=1}^{n}\left(2 d_{j}-1\right) ! !}
\end{aligned}
$$

Note that Faber's identity (3) and all of the above identities (4)-(9) are compatible with the string and dilaton equations, so $d_{j} \geq 2$ may be assumed when proving these identities. We have checked the identities of conjecture 2.2 and conjecture 2.5 for all $g \leq 20$ by computer.

Now we give a proof of Conjecture 2.5 for $n=1$.

$$
\frac{g-1}{2^{2 g}(2 g+1) ! !}=\left\langle\tau_{g+1} \tau_{2 g-2}\right\rangle_{g}-\left\langle\tau_{3 g-2}\right\rangle_{g}+\sum_{j=0}^{2 g-4}(-1)^{j}\left\langle\tau_{j} \tau_{g+1}\right\rangle\left\langle\tau_{2 g-4-j}\right\rangle .
$$

In the last sum of the right hand side, replace $j$ by $3 h-g-2$. We need to prove

$$
\sum_{h=1}^{g} \frac{(-1)^{g-h}}{24^{g-h}(g-h) !}\left\langle\tau_{3 h-g-2} \tau_{g+1}\right\rangle_{h}=\frac{g-1}{2^{2 g}(2 g+1) ! !}+\left\langle\tau_{3 g-2}\right\rangle_{g}
$$

Apply the string equation twice, we have

$$
\begin{aligned}
& \sum_{h=1}^{g} \frac{(-1)^{g-h}}{24^{g-h}(g-h) !}\left\langle\tau_{3 h-g-2} \tau_{g+1}\right\rangle_{h} \\
& \quad=\sum_{h=1}^{g} \frac{(-1)^{g-h}}{24^{g-h}(g-h) !}\left(\left\langle\tau_{0} \tau_{3 h-g-1} \tau_{g+1}\right\rangle_{h}-\left\langle\tau_{3 h-g-1} \tau_{g}\right\rangle_{h}\right) \\
& =\sum_{h=1}^{g} \frac{(-1)^{g-h}}{24^{g-h}(g-h) !}\left(\left\langle\tau_{0} \tau_{3 h-g-1} \tau_{g+1}\right\rangle_{h}-\left\langle\tau_{0} \tau_{3 h-g} \tau_{g}\right\rangle_{h}+\left\langle\tau_{3 h-g} \tau_{g-1}\right\rangle_{h}\right) .
\end{aligned}
$$

Here we need some knowledge of $n$-point functions such as in the papers [5, 9, 19]. Since $\sum_{h=0}^{g} \frac{(-1)^{g-h}}{24^{g-h}(g-h) !}\left\langle\tau_{0} \tau_{3 h-g-1} \tau_{g+1}\right\rangle_{h}$ and $\sum_{h=0}^{g} \frac{(-1)^{g-h}}{24^{g-h}(g-h) !}\left\langle\tau_{0} \tau_{3 h-g} \tau_{g}\right\rangle_{h}$ are 
respectively the coefficient of $y^{2 g-1} z^{g+1}$ and $y^{2 g} z^{g}$ in

$$
\exp \left(\frac{z^{3}}{24}\right) \sum_{k \geq 0} \frac{k !}{(2 k+1) !}\left(\frac{1}{2} y z(y+z)\right)^{k}
$$

we have

$$
\begin{aligned}
\sum_{h=1}^{g} \frac{(-1)^{g-h}}{24^{g-h}(g-h) !}\left\langle\tau_{0} \tau_{3 h-g-1} \tau_{g+1}\right\rangle_{h} & =\frac{g !}{(2 g+1) !} \cdot \frac{g}{2^{g}} \\
\sum_{h=1}^{g} \frac{(-1)^{g-h}}{24^{g-h}(g-h) !}\left\langle\tau_{0} \tau_{3 h-g} \tau_{g}\right\rangle_{h} & =\frac{g !}{(2 g+1) !} \cdot \frac{1}{2^{g}} .
\end{aligned}
$$

Moreover, it has been proved in $[5,9]$ that

$$
\sum_{h=1}^{g} \frac{(-1)^{g-h}}{24^{g-h}(g-h) !}\left\langle\tau_{3 h-g} \tau_{g-1}\right\rangle_{h}=\frac{1}{24^{g} g !}
$$

so we conclude the proof.

Both of Theorem 2.1 and Theorem 2.3 follow from our recently obtained $n$-point functions for intersection numbers.

Definition 2.7. We call the following generating function

$$
F\left(x_{1}, \ldots, x_{n}\right)=\sum_{g=0}^{\infty} \sum_{\sum d_{j}=3 g-3+n}\left\langle\tau_{d_{1}} \cdots \tau_{d_{n}}\right\rangle_{g} \prod_{j=1}^{n} x_{j}^{d_{j}}
$$

the $n$-point function.

Note that the left hand side of identity (4) in Theorem 2.1 is

$$
\left[F\left(y,-y, x_{1}, \ldots, x_{n}\right)\right]_{y^{2 g-2} \prod_{j=1}^{n} x_{j}^{d_{j}}}
$$

which is the coefficient of the monomial $y^{2 g-2} \prod_{j=1}^{n} x_{j}^{d_{j}}$ in the special $(n+2)$-point function $F\left(y,-y, x_{1}, \ldots, x_{n}\right)$.

It's not an easy task to get explicit formulae for $n$-point functions [2]. Okounkov [22] has obtained a marvelous analytic formula for $n$-point functions, however it seems very difficult to extract information of coefficients from this analytic formula. What's more interesting is to find some well organized series expansion for the $n$-point function.

We introduce the following "normalized" $n$-point function

$$
G\left(x_{1}, \ldots, x_{n}\right)=\exp \left(\frac{-\sum_{j=1}^{n} x_{j}^{3}}{24}\right) \cdot F\left(x_{1}, \ldots, x_{n}\right) .
$$

Theorem 2.8. [19] For $n \geq 2$,

$$
G\left(x_{1}, \ldots, x_{n}\right)=\sum_{r, s \geq 0} \frac{(2 r+n-3) ! !}{4^{s}(2 r+2 s+n-1) ! !} P_{r}\left(x_{1}, \ldots, x_{n}\right) \Delta\left(x_{1}, \ldots, x_{n}\right)^{s}
$$


where $P_{r}$ and $\Delta$ are homogeneous symmetric polynomials defined by

$$
\begin{aligned}
\Delta\left(x_{1}, \ldots, x_{n}\right) & =\frac{\left(\sum_{j=1}^{n} x_{j}\right)^{3}-\sum_{j=1}^{n} x_{j}^{3}}{3}, \\
P_{r}\left(x_{1}, \ldots, x_{n}\right) & =\left(\frac{1}{2 \sum_{j=1}^{n} x_{j}} \sum_{\underline{n}=I \amalg J}\left(\sum_{i \in I} x_{i}\right)^{2}\left(\sum_{i \in J} x_{i}\right)^{2} G\left(x_{I}\right) G\left(x_{J}\right)\right)_{3 r+n-3} \\
& =\frac{1}{2 \sum_{j=1}^{n} x_{j}} \sum_{\underline{n}=I \amalg J}\left(\sum_{i \in I} x_{i}\right)^{2}\left(\sum_{i \in J} x_{i}\right)^{2} \sum_{r^{\prime}=0}^{r} G_{r^{\prime}}\left(x_{I}\right) G_{r-r^{\prime}}\left(x_{J}\right),
\end{aligned}
$$

where $I, J \neq \emptyset, \underline{n}=\{1,2, \ldots, n\}$ and $G_{g}\left(x_{I}\right)$ denotes the degree $3 g+|I|-3$ homogeneous component of the normalized $|I|$-point function $G\left(x_{k_{1}}, \ldots, x_{k_{|I|}}\right)$, where $k_{j} \in I$.

The above formula generalizes Dijkgraaf's two-point function [5] and Zagier's threepoint function [25] obtained more than ten years ago.

Let's turn to the normalized special $(n+2)$-point function,

$$
G\left(y,-y, x_{1}, \ldots, x_{n}\right)=\exp \left(\frac{-\sum_{j=1}^{n} x_{j}^{3}}{24}\right) \cdot F\left(y,-y, x_{1}, \ldots, x_{n}\right) .
$$

We have the following theorem [19] about the coefficients of $G\left(y,-y, x_{1}, \ldots, x_{n}\right)$, which is just a reformulation of Theorem 2.3 and Theorem 2.1.

Theorem 2.9. Let $g \geq 0$ and $n \geq 1$. We have

(1) Let $K>g, d_{j} \geq 0$ and $\sum_{j=1}^{n} d_{j}=3 g-1+n-2 K$. Then

$$
\left[G\left(y,-y, x_{1}, \ldots, x_{n}\right)\right]_{y^{2 K}} \prod_{j=1}^{n} x_{j}^{d_{j}}=0 .
$$

(2) Let $d_{j} \geq 1$ and $\sum_{j=1}^{n} d_{j}=g-1+n$. Then

$$
\left[G\left(y,-y, x_{1}, \ldots, x_{n}\right)\right]_{y^{2 g} \prod_{j=1}^{n} x_{j}^{d_{j}}}=\frac{(2 g+n-1) !}{4^{g}(2 g+1) ! \cdot \prod_{j=1}^{n}\left(2 d_{j}-1\right) ! !} .
$$

\section{Vanishing phenomenon of intersection numbers}

We investigate a sort of vanishing phenomenon of intersection numbers and seek further clarification for our simplified version of Faber's conjecture. First we state a generalization of Theorem 2.3.

Conjecture 3.1. Let $K>g, d_{j} \geq 0$ and $\Lambda$ be a monomial of $\lambda$ classes. Then

$$
\sum_{j=0}^{2 K}(-1)^{j}\left\langle\tau_{2 K-j} \tau_{j} \Lambda \prod_{j=1}^{n} \tau_{d_{j}}\right\rangle_{g}=0
$$


Take $\Lambda=\lambda_{1}$ in Conjecture 3.1. Since $\lambda_{1}=\operatorname{ch}_{1}(\mathbb{E})$, we have

$$
\begin{aligned}
& 0=12 \sum_{j=0}^{2 K}(-1)^{j}\left\langle\tau_{2 K-j} \tau_{j} \lambda_{1} \prod_{j=1}^{n} \tau_{d_{j}}\right\rangle_{g} \\
& =-\sum_{j=0}^{2 K}(-1)^{j}\left(\left\langle\tau_{2 K-j+1} \tau_{j} \prod_{j=1}^{n} \tau_{d_{j}}\right\rangle_{g}+\left\langle\tau_{2 K-j} \tau_{j+1} \prod_{j=1}^{n} \tau_{d_{j}}\right\rangle_{g}\right) \\
& \quad+\sum_{\underline{n}=I \amalg J} \sum_{j=0}^{2 K}(-1)^{j}\left\langle\tau_{j} \tau_{0} \prod_{i \in I} \tau_{d_{i}}\right\rangle_{g^{\prime}}\left\langle\tau_{2 K-j} \tau_{0} \prod_{i \in J} \tau_{d_{i}}\right\rangle_{g-g^{\prime}} \\
& =\sum_{\underline{n}=I \amalg J} \sum_{j=0}^{2 K}(-1)^{j}\left\langle\tau_{j} \tau_{0} \prod_{i \in I} \tau_{d_{i}}\right\rangle_{g^{\prime}}\left\langle\tau_{2 K-j} \tau_{0} \prod_{i \in J} \tau_{d_{i}}\right\rangle_{g-g^{\prime}}-2\left\langle\tau_{2 K+1} \tau_{0} \prod_{j=1}^{n} \tau_{d_{j}}\right\rangle_{g} .
\end{aligned}
$$

We have used Theorem 2.3 in the above equations. In fact, we have identities much more general than the above equation.

\section{Conjecture 3.2. We have}

(1) Let $K \geq g, r, s \geq 0, d_{j} \geq 0$ and $\sum_{j=1}^{n} d_{j}=3 g+n-2 K-r-s-2$. Then

$$
\begin{aligned}
&\left\langle\tau_{2 K+r+1} \tau_{s} \prod_{j=1}^{n} \tau_{d_{j}}\right\rangle_{g}+\left\langle\tau_{2 K+s+1} \tau_{r} \prod_{j=1}^{n} \tau_{d_{j}}\right\rangle_{g} \\
&=\sum_{\underline{n}=I \amalg} \sum_{j=0}^{2 K}(-1)^{j}\left\langle\tau_{j} \tau_{r} \prod_{i \in I} \tau_{d_{i}}\right\rangle_{g^{\prime}}\left\langle\tau_{2 K-j} \tau_{s} \prod_{i \in J} \tau_{d_{i}}\right\rangle_{g-g^{\prime}}
\end{aligned}
$$

(2) Let $r, s \geq 0, d_{j} \geq 1$ and $\sum_{j=1}^{n} d_{j}=g+n-r-s$. Then

$$
\begin{aligned}
\frac{1}{(2 r+1) ! !(2 s+1) ! !} & \frac{(2 g-1+n) !}{4^{g}(2 g-1) ! \prod_{j=1}^{n}\left(2 d_{j}-1\right) ! !} \\
= & \left\langle\tau_{2 g+r-1} \tau_{s} \prod_{j=1}^{n} \tau_{d_{j}}\right\rangle_{g}+\left\langle\tau_{2 g+s-1} \tau_{r} \prod_{j=1}^{n} \tau_{d_{j}}\right\rangle_{g} \\
& \quad-\sum_{\underline{n}=I \amalg J} \sum_{j=0}^{2 g-2}(-1)^{j}\left\langle\tau_{j} \tau_{r} \prod_{i \in I} \tau_{d_{i}}\right\rangle_{g^{\prime}}\left\langle\tau_{2 g-2-j} \tau_{s} \prod_{i \in J} \tau_{d_{i}}\right\rangle_{g-g^{\prime}}
\end{aligned}
$$

Identities in Conjecture 3.2 have the same structures as results in the last section, we believe there is a uniform way to prove these conjectural identities. 
Taking $\Lambda=\operatorname{ch}_{2 r+1}(\mathbb{E})$ in Conjecture 3.1 and $K>g$, we have

$$
\begin{gathered}
0=\frac{(2 r+2) !}{B_{2 r+2}} \sum_{j=0}^{2 K}(-1)^{j}\left\langle\tau_{2 K-j} \tau_{j} \operatorname{ch}_{2 r+1}(\mathbb{E}) \prod_{i=1}^{n} \tau_{d_{i}}\right\rangle_{g} \\
=-\sum_{j=0}^{2 K}(-1)^{j}\left(\left\langle\tau_{2 K-j+2 r+1} \tau_{j} \prod_{i=1}^{n} \tau_{d_{i}}\right\rangle_{g}+\left\langle\tau_{2 K-j} \tau_{j+2 r+1} \prod_{i=1}^{n} \tau_{d_{i}}\right\rangle_{g}\right) \\
+\sum_{\underline{n}=I \amalg J} \sum_{i=0}^{2 r} \sum_{j=0}^{2 K}(-1)^{i+j}\left\langle\tau_{i} \tau_{j} \prod_{t \in I} \tau_{d_{t}}\right\rangle_{g^{\prime}}\left\langle\tau_{2 r-i} \tau_{2 K-j} \prod_{t \in J} \tau_{d_{t}}\right\rangle_{g-g^{\prime}} \\
\quad=-2 \sum_{i=0}^{2 r}(-1)^{i}\left\langle\tau_{2 K+2 r+1-i} \tau_{i} \prod_{j=1}^{n} \tau_{d_{j}}\right\rangle_{g} \\
+\sum_{\underline{n}=I \amalg J} \sum_{i=0}^{2 r} \sum_{j=0}^{2 K}(-1)^{i+j}\left\langle\tau_{i} \tau_{j} \prod_{t \in I} \tau_{d_{t}}\right\rangle_{g^{\prime}}\left\langle\tau_{2 r-i} \tau_{2 K-j} \prod_{t \in J} \tau_{d_{t}}\right\rangle_{g-g^{\prime}}
\end{gathered}
$$

It's not difficult to see that conjecture 3.2(1) implies Conjecture 3.1 in the case $\Lambda=\operatorname{ch}_{2 r+1}(\mathbb{E})$.

Now we present a hierarchy of conjectural identities of intersection numbers, which provide further insights to Faber's conjecture.

Conjecture 3.3. For $m \geq 2$, we have

(1) Let $K \geq g+\left\lfloor\frac{m}{2}\right\rfloor-1, r_{p} \geq 0, d_{j} \geq 0$ and $\sum_{j=1}^{n} d_{j}=3 g+n-2 K-\sum_{p=1}^{m} r_{p}+$ $m-4$. Then

$$
\begin{aligned}
\left\langle\tau_{2 K+2} \prod_{p=1}^{m} \tau_{r_{p}} \prod_{j=1}^{n} \tau_{d_{j}}\right\rangle_{g} & =\sum_{j=1}^{n}\left\langle\tau_{d_{1}} \ldots \tau_{d_{j-1}} \tau_{d_{j}+2 K+1} \tau_{d_{j+1}} \ldots \tau_{d_{n}} \prod_{p=1}^{m} \tau_{r_{p}}\right\rangle_{g} \\
& -\sum_{\underline{n}=I \amalg} \sum_{j=0}^{2 K}(-1)^{j}\left\langle\tau_{j} \prod_{p=1}^{m} \tau_{r_{p}} \prod_{i \in I} \tau_{d_{i}}\right\rangle_{g^{\prime}}\left\langle\tau_{2 K-j} \prod_{i \in J} \tau_{d_{i}}\right\rangle_{g-g^{\prime}} .
\end{aligned}
$$

(2) Let $K=g+\left\lfloor\frac{m}{2}\right\rfloor-2, d_{j} \geq 1$ and $\sum_{j=1}^{n} d_{j}=g+n-\sum_{p} r_{p}+m-2\left\lfloor\frac{m}{2}\right\rfloor$. Define

$$
C\left(g, m, r_{p}\right)= \begin{cases}\left(\sum_{p} 2 r_{p}+m\right)\left(g+\frac{m-3}{2}\right) & \text { if } m \text { is odd } \\ 1 & \text { if } m \text { is even }\end{cases}
$$

Then we have

$$
\begin{aligned}
\frac{C\left(g, m, r_{p}\right)}{\prod_{p=1}^{m}\left(2 r_{p}+1\right) ! !} & \frac{(2 g-3+n+m) !}{4^{g}(2 g-3+m) ! \prod_{j=1}^{n}\left(2 d_{j}-1\right) ! !} \\
=\left\langle\tau_{2 K+2} \prod_{p=1}^{m} \tau_{r_{p}} \prod_{j=1}^{n} \tau_{d_{j}}\right\rangle_{g}-\sum_{j=1}^{n}\left\langle\tau_{d_{1}} \ldots \tau_{d_{j-1}} \tau_{d_{j}+2 K+1} \tau_{d_{j+1}} \ldots \tau_{d_{n}} \prod_{p=1}^{m} \tau_{r_{p}}\right\rangle_{g} & \\
& \quad+\sum_{\underline{n}=I \amalg J} \sum_{j=0}^{2 K}(-1)^{j}\left\langle\tau_{j} \prod_{p=1}^{m} \tau_{r_{p}} \prod_{i \in I} \tau_{d_{i}}\right\rangle_{g^{\prime}}\left\langle\tau_{2 K-j} \prod_{i \in J} \tau_{d_{i}}\right\rangle_{g-g^{\prime}} .
\end{aligned}
$$

If $m$ is odd, we require $r_{p} \geq 1$ in the above identity. 
Conjecture 3.4. For $m \geq 2$, we have

(1) Let $K \geq g+\left\lfloor\frac{m-1}{2}\right\rfloor, s \geq 0, r_{p} \geq 0, d_{j} \geq 0$ and $\sum_{j=1}^{n} d_{j}=3 g+n-2 K-s-$ $\sum_{p=1}^{m} r_{p}+m-3$. Then

$\left\langle\tau_{2 K+s+1} \prod_{p=1}^{m} \tau_{r_{p}} \prod_{j=1}^{n} \tau_{d_{j}}\right\rangle_{g}$

$$
=\sum_{\underline{n}=I} \sum_{J}^{2 K}(-1)^{j}\left\langle\tau_{j} \prod_{p=1}^{m} \tau_{r_{p}} \prod_{i \in I} \tau_{d_{i}}\right\rangle_{g^{\prime}}\left\langle\tau_{2 K-j} \tau_{s} \prod_{i \in J} \tau_{d_{i}}\right\rangle_{g-g^{\prime}} .
$$

(2) Let $K=g+\left\lfloor\frac{m-1}{2}\right\rfloor-1, d_{j} \geq 1$ and $\sum_{j=1}^{n} d_{j}=g+n-s-\sum_{p} r_{p}+m-$ $2\left\lfloor\frac{m-1}{2}\right\rfloor-1$. Define

$$
C\left(g, m, s, r_{p}\right)= \begin{cases}\left(\sum_{p} 2 r_{p}-2 s+m-1\right)\left(g+\frac{m}{2}-1\right) & \text { if } m \text { is even, } \\ 1 & \text { if } m \text { is odd. }\end{cases}
$$

Then we have

$$
\begin{aligned}
\frac{C\left(g, m, s, r_{p}\right)}{(2 s+1) ! ! \prod_{p=1}^{m}\left(2 r_{p}+1\right) ! !} & \cdot \frac{(2 g-2+n+m) !}{4^{g}(2 g-2+m) ! \prod_{j=1}^{n}\left(2 d_{j}-1\right) ! !} \\
& =\left\langle\tau_{2 K+s+1} \prod_{p=1}^{m} \tau_{r_{p}} \prod_{j=1}^{n} \tau_{d_{j}}\right\rangle_{g} \\
& -\sum_{\underline{n}=I} \sum_{J}^{2 K}(-1)^{j}\left\langle\tau_{j} \prod_{p=1}^{m} \tau_{r_{p}} \prod_{i \in I} \tau_{d_{i}}\right\rangle_{g^{\prime}}\left\langle\tau_{2 K-j} \tau_{s} \prod_{i \in J} \tau_{d_{i}}\right\rangle_{g-g^{\prime}} .
\end{aligned}
$$

If $m$ is even, we require $s \geq 1$ and $r_{p} \geq 1$ in the above identity.

The following conjecture generalizes results of [19].

Conjecture 3.5. For $m, l \geq 2$, we have

(1) Let $K>2 g+m+l-4, r_{p}, s_{p} \geq 0, d_{j} \geq 0$ and $\sum_{j=1}^{n} d_{j}=3 g+n+m+l-$ $K-\sum_{p=1}^{m} r_{p}-\sum_{p=1}^{l} s_{p}-4$. Then

$$
\sum_{\underline{n}=I \amalg} \sum_{j=0}^{K}(-1)^{j}\left\langle\tau_{j} \prod_{p=1}^{m} \tau_{r_{p}} \prod_{t \in I} \tau_{d_{t}}\right\rangle\left\langle\tau_{K-j} \prod_{p=1}^{l} \tau_{s_{p}} \prod_{t \in J} \tau_{d_{t}}\right\rangle=0 .
$$

(2) Let $K=2 g+m+l-4, d_{j} \geq 1$ and $\sum_{j=1}^{n} d_{j}=g+n-\sum_{p} r_{p}-\sum_{p} s_{p}$. Then

$$
\begin{aligned}
& \sum_{\underline{n}=I \amalg} \sum_{J}^{K}(-1)^{j}\left\langle\tau_{j} \prod_{p=1}^{m} \tau_{r_{p}} \prod_{t \in I} \tau_{d_{t}}\right\rangle\left\langle\tau_{K-j} \prod_{p=1}^{l} \tau_{s_{p}} \prod_{t \in J} \tau_{d_{t}}\right\rangle \\
& =\frac{1}{\prod_{p=1}^{m}\left(2 r_{p}+1\right) ! ! \prod_{p=1}^{l}\left(2 s_{p}+1\right) ! !} \cdot \frac{(-1)^{m}(2 g+n+m+l-3) !}{4^{g}(2 g+m+l-3) ! \prod_{j=1}^{n}\left(2 d_{j}-1\right) ! !} .
\end{aligned}
$$




\section{Denominators of intersection numbers}

Let $\operatorname{denom}(r)$ denotes the denominator of a rational number $r$ in reduced form (coprime numerator and denominator, positive denominator). We define

$$
D_{g, n}=l \mathrm{~cm}\left\{\operatorname{denom}\left(\int_{\overline{\mathcal{M}}_{g, n}} \psi_{1}^{d_{1}} \cdots \psi_{n}^{d_{n}}\right) \mid \sum_{i=1}^{n} d_{i}=3 g-3+n\right\}
$$

and for $g \geq 2$,

$$
\mathcal{D}_{g}=\operatorname{lcm}\left\{\operatorname{denom}\left(\int_{\overline{\mathcal{M}}_{g}} \kappa_{a_{1}} \cdots \kappa_{a_{m}}\right) \mid \sum_{i=1}^{m} a_{m}=3 g-3\right\}
$$

where $l \mathrm{~cm}$ is the abbreviation of least common multiple.

We proved in our previous paper [18] that $D_{g, n} \mid \mathcal{D}_{g}$ and $D_{g, n}=\mathcal{D}_{g}$ for $n \geq 3 g-3$.

Now we present the conjectural exact values of $\mathcal{D}_{g}$.

Conjecture 4.1. Let $p$ denotes a prime number and $g \geq 2$. Let $\operatorname{ord}(p, n)$ denotes the maximum integer such that $p^{\operatorname{ord}(p, n)} \mid n$. Then

(1) $\operatorname{ord}\left(2, \mathcal{D}_{g}\right)=3 g+\operatorname{ord}(2, g !)$,

(2) $\operatorname{ord}\left(3, \mathcal{D}_{g}\right)=g+\operatorname{ord}(3, g !)$,

(3) $\operatorname{ord}\left(p, \mathcal{D}_{g}\right)=\left\lfloor\frac{2 g}{p-1}\right\rfloor$ for $p \geq 5$, where $\lfloor x\rfloor$ denotes the maximum integer that is not larger than $x$.

We order all Witten-Kontsevich tau functions of given genus $g$ by the following lexicographical rule,

$$
\left\langle\tau_{d_{1}} \cdots \tau_{d_{n}}\right\rangle_{g} \prec\left\langle\tau_{k_{1}} \cdots \tau_{k_{m}}\right\rangle_{g}
$$

if $n<m$ or $n=m$ and there exists some $i$, such that $d_{j}=k_{j}$ for $j<i$ and $d_{i}<k_{i}$.

If $5 \leq p \leq 2 g+1$ is a prime number, then the smallest tau function of genus $g$ in the above lexicographical order that satisfies $\operatorname{ord}\left(p, \operatorname{denom}\left\langle\tau_{d_{1}} \cdots \tau_{d_{n}}\right\rangle_{g}\right)=\left\lfloor\frac{2 g}{p-1}\right\rfloor$ is

$$
\underbrace{\left\langle\tau_{\frac{p-1}{2}} \cdots \tau_{\frac{p-1}{2}}\right.}_{\left\lfloor\frac{2 g}{p-1}\right\rfloor} \tau_{d}\rangle_{g}
$$

where $d+\frac{p-1}{2}\left\lfloor\frac{2 g}{p-1}\right\rfloor=3 g-2+\left\lfloor\frac{2 g}{p-1}\right\rfloor$.

We have checked Conjecture 4.1 for all $g \leq 20$ by a computer.

Corollary 4.2. We have $D_{g, n}=\mathcal{D}_{g}$ for $n \geq\left\lfloor\frac{g}{2}\right\rfloor+1$.

Corollary 4.3. Let $\mathcal{D}_{0}=1$ and $\mathcal{D}_{1}=24$, then $\mathcal{D}_{g} \mathcal{D}_{h} \mid \mathcal{D}_{g+h}$, for $g, h \geq 0$.

Note that $\left\lfloor\frac{g}{2}\right\rfloor+1$ is just the number of codimension one boundary strata of $\overline{\mathcal{M}}_{g}$, we don't know whether this has any implications.

We remark that that $\mathcal{D}_{g}$ does not control the denominators of general Hodge integrals, since we have ord $\left(5, \operatorname{denom}\left\langle\tau_{19} \lambda_{9}\right\rangle_{10}\right)=6>\operatorname{ord}\left(5, \mathcal{D}_{10}\right)$.

Let $\mathcal{S}_{g}$ be the least common multiple of $\left\{\left|A u t\left(\Sigma_{g}\right)\right|\right\}$, where $\Sigma_{g}$ takes over all stable curves of genus $g$. By arranging components of stable curves in a most symmetric way, it's not difficult to see that

$$
\begin{aligned}
& \operatorname{ord}\left(2, \mathcal{S}_{g}\right) \geq 2 g+\lfloor g / 2\rfloor+\lfloor\lfloor g / 2\rfloor / 2\rfloor+\lfloor\lfloor\lfloor g / 2\rfloor / 2\rfloor / 2\rfloor+\cdots \\
& \operatorname{ord}\left(p, \mathcal{S}_{g}\right) \geq k+\lfloor k / p\rfloor+\lfloor\lfloor k / p\rfloor / p\rfloor+\lfloor\lfloor\lfloor k / p\rfloor / p\rfloor / p\rfloor+\cdots, \text { if prime } p \geq 3,
\end{aligned}
$$


where $k=\left\lfloor\frac{2 g}{p-1}\right\rfloor$. We conjecture that the above relations are actually equalities giving exact values of $\mathcal{S}_{g}$.

It's easy to see that $\operatorname{ord}\left(2, \mathcal{D}_{g}\right)>\operatorname{ord}\left(2, \mathcal{S}_{g}\right), \operatorname{ord}\left(3, \mathcal{D}_{g}\right) \geq \operatorname{ord}\left(3, \mathcal{S}_{g}\right)$ and for $p \geq 5$, $\operatorname{ord}\left(p, \mathcal{D}_{g}\right) \leq \operatorname{ord}\left(p, \mathcal{S}_{g}\right)$. Roughly speaking, this means that top intersections of kappa classes fail to detect all singularities on the orbifold $\overline{\mathcal{M}}_{g}$.

\section{Numerical properties of intersection numbers}

From Okounkov's analytic formula of $n$-point functions [22], we have

$$
F\left(x_{1}, \ldots, x_{n}\right)=\sum_{g=0}^{\infty} \sum_{\sum d_{j}=3 g-3+n}\left\langle\tau_{d_{1}} \cdots \tau_{d_{n}}\right\rangle_{g} \prod_{j=1}^{n} x_{j}^{d_{j}}<\infty .
$$

for arbitrary positive real numbers $x_{i}$. So $\left\langle\tau_{d_{1}} \cdots \tau_{d_{n}}\right\rangle_{g}$ decreases very rapidly when $g$ increases. In this section, we will discuss a kind of multinomial-type property for intersection numbers.

Conjecture 5.1. Let $\Lambda$ be a monomial of the form $\lambda_{1}^{k_{1}} \cdots \lambda_{g}^{k_{g}}$. Then for $\sum_{j=1}^{n} d_{j}=$ $3 g-3+n-\sum_{j=1}^{g} k_{j} \cdot j$ and $d_{1}<d_{2}$, we have

$$
\int_{\overline{\mathcal{M}}_{g, n}} \psi_{1}^{d_{1}} \psi_{2}^{d_{2}} \cdots \psi_{n}^{d_{n}} \Lambda \leq \int_{\overline{\mathcal{M}}_{g, n}} \psi_{1}^{d_{1}+1} \psi_{2}^{d_{2}-1} \cdots \psi_{n}^{d_{n}} \Lambda .
$$

Namely the more evenly $3 g-3+n$ be distributed among indices, the larger the value of Hodge integrals.

From the argument of Proposition 5.1 of [18], we see it's enough to check only those Hodge integrals with $d_{3} \geq 2, \ldots, d_{n} \geq 2$. We have checked Conjecture 5.1 in various cases.

For $\Lambda=1$, namely in the case of tau functions, we have checked Conjecture 5.1 for $g \leq 20$. Moreover, for $n=2$, we have checked all $g \leq 1000$ (using Dijkgraaf's 2-point function); for $n=3$, we have checked all $g \leq 100$ (using Zagier's 3-point function).

For $\Lambda=\lambda_{g}$, we have the $\lambda_{g}$ theorem proved by Faber and Pandharipande [8],

$$
\int_{\overline{\mathcal{M}}_{g, n}} \psi_{1}^{d_{1}} \cdots \psi_{n}^{d_{n}} \lambda_{g}=\left(\begin{array}{c}
2 g+n-3 \\
d_{1}, \ldots, d_{n}
\end{array}\right) \frac{2^{2 g-1}-1}{2^{2 g-1}} \frac{\left|B_{2 g}\right|}{(2 g) !} .
$$

For $\Lambda=\lambda_{g} \lambda_{g-1}$, we have the Faber's conjecture.

For $\Lambda=\lambda_{1}^{k_{1}} \cdots \lambda_{g}^{k_{g}}$, where $\sum_{i=1}^{g} i k_{i}=3 g-3$, we can use the same argument as Proposition 5.1 in [18].

For $\Lambda=\lambda_{g-1}$, there is a closed formula for Hodge integrals with $\lambda_{g-1}$ class in [12], we have checked the case of two-point Hodge integrals for $g \leq 100$.

Based on a large amount of experiment, we speculate that the following generalization of Conjecture 5.1 should be true.

Conjecture 5.2. Consider the following general intersection numbers,

$$
\left\langle\tau_{\underline{d}} \kappa_{\underline{a}} \lambda_{\underline{b}}\right\rangle_{g, n}:=\int_{\overline{\mathcal{M}}_{g, n}} \psi_{1}^{d_{1}} \cdots \psi_{n}^{d_{n}} \kappa_{a_{1}} \cdots \kappa_{a_{m}} \lambda_{b_{1}} \cdots \lambda_{b_{k}}
$$


where the indices $\left\{d_{j}, a_{j}, b_{j}\right\}$ are nonnegative integers. If $p<q$ we have

$$
\begin{aligned}
&\left\langle\tau_{p} \tau_{q} \tau_{\underline{d}} \kappa_{\underline{a}} \lambda_{\underline{b}}\right\rangle_{g, n} \leq\left\langle\tau_{p+1} \tau_{q-1} \tau_{\underline{d}} \kappa_{\underline{a}} \lambda_{\underline{b}}\right\rangle_{g, n}, \\
&\left\langle\kappa_{p} \kappa_{q} \tau_{\underline{d}} \kappa_{\underline{a}} \lambda_{\underline{b}}\right\rangle_{g, n} \leq\left\langle\kappa_{p+1} \kappa_{q-1} \tau_{\underline{d}} \kappa_{a} \lambda_{\underline{b}}\right\rangle_{g, n}, \\
&\left\langle\lambda_{p} \lambda_{q} \tau_{\underline{d}} \kappa_{\underline{a}} \lambda_{\underline{b}}\right\rangle_{g, n} \leq\left\langle\lambda_{p+1} \lambda_{q-1} \tau_{\underline{d}} \kappa_{a} \lambda_{\underline{b}}\right\rangle_{g, n} .
\end{aligned}
$$

We make some remarks about Conjecture 5.2. First recall the definition of Schur polynomials. Let $E$ be a vector bundle of rank $r$ on a projective variety $X$ and $\mu$ be a partition of integer $n$ into integers $\leq r$,

$$
r \geq \mu_{1} \geq \mu_{2} \geq \cdots \geq \mu_{n} \geq 0
$$

Define the Schur polynomials $s_{\mu}(E)$ by

$$
s_{\mu}(E)=\operatorname{det}\left(c_{\mu_{i}+j-i}(E)\right)_{1 \leq i, j \leq n} .
$$

So $s_{\mu}(E)$ is a polynomial in Chern classes of $E$ with weighted degree $n$. If $E$ is ample, then we know that $s_{\mu}(E)$ behave numerical positively on $X$.

However the Hodge bundle $\mathbb{E}$ on $\overline{\mathcal{M}}_{g}$ is not ample, only its $\operatorname{determinant} \operatorname{det}(\mathbb{E})$ is semi-ample. If $\mu=\left(b_{2}, b_{1}, 0, \ldots, 0\right)$, then $s_{\mu}(\mathbb{E})=\lambda_{b_{2}} \lambda_{b_{1}}-\lambda_{b_{2}+1} \lambda_{b_{1}-1}$. So conjecture $5.2(3)$ essentially says that Schur polynomials of the form $s_{\left(b_{2}, b_{1}\right)}(\mathbb{E})$ behave numerical positively on the moduli space of curves.

There are several natural consequences from Conjecture 5.2. For example, we could get simple lower and upper bounds for intersections of kappa classes, the so called higher Weil-Petersson volumes of the moduli space of curves [16].

Corollary 5.3. Let $a_{j} \geq 0, \sum_{j=1}^{m} a_{j}=3 g-3+n$ and $g \geq 1$. We have

$$
\frac{(2 g-2+n)^{m-1}}{24^{g} \cdot g !} \leq\left\langle\kappa_{a_{1}} \cdots \kappa_{a_{m}}\right\rangle_{g, n} \leq \frac{\left\langle\kappa_{1}^{3 g-3+n}\right\rangle_{g, n}}{(2 g-2+n)^{3 g-3+n-m}} .
$$

In particular, we get a simple lower bound for Weil-Petersson volumes

$$
\left\langle\kappa_{1}^{3 g-3+n}\right\rangle_{g, n} \geq \frac{(2 g-2+n)^{3 g-4+n}}{24^{g} \cdot g !} .
$$

Proof. Since $\kappa_{0}=2 g-2+n$ in $\mathcal{R}^{0}\left(\overline{\mathcal{M}}_{g, n}\right)$, we have

$$
\left\langle\kappa_{a_{1}} \cdots \kappa_{a_{m}}\right\rangle_{g, n} \geq\left\langle\kappa_{3 g-3+n} \kappa_{0}^{m-1}\right\rangle_{g, n}=\frac{(2 g-2+n)^{m-1}}{24^{g} \cdot g !} .
$$

The other inequality can be proved similarly.

Corollary 5.4. For $d_{j} \geq 0$ and $\sum_{j=1}^{n} d_{j}=3 g-3+n$,

$$
\left\langle\tau_{d_{1}} \cdots \tau_{d_{n}}\right\rangle_{g} \geq \frac{1}{24^{g} \cdot g !} .
$$




\section{References}

[1] E. Arbarello and M. Cornalba, Combinatorial and Algebro-Geometric cohomology classes on the Moduli Spaces of Curves, J. Algebraic Geometry, 5 (1996), 705-709.

[2] E. Brézin and S. Hikami, Vertices from replica in a random matrix theory, math-ph/0704.2044.

[3] L. Chen, Y. Li and K. Liu, Localization, Hurwitz numbers and the witten conjecture, preprint, math.AG/0609263.

[4] R. Dijkgraaf, Intersection Theory, Integrable Hierarchies and Topological Field Theory, New symmetry principles in quantum field theory (Cargse, 1991), 95-158, NATO Adv. Sci. Inst. Ser. B Phys., 295, Plenum, New York, 1992.

[5] C. Faber, A non-vanishing result for the tautological ring of $\mathcal{M}_{g}$, math/9711219 (November 1997).

[6] _ A conjectural description of the tautological ring of the moduli space of curves. In Moduli of curves and abelian varieties, Aspects Math., E33, Vieweg, Braunschweig, Germany, 1999. 109-129.

[7] _ Algorithms for computing intersection numbers on moduli spaces of curves, with an application to the class of the locus of Jacobians, in New Trends in Algebraic Geometry (K. Hulek, F. Catanese, C. Peters and M. Reid, eds.), 93-109, Cambridge University Press, 1999.

[8] C. Faber, R. Pandharipande, Hodge integrals, partition matrices, and the $\lambda_{g}$ conjecture, Ann. Math. 156 (2002), 97-124.

[9] Logarithmic series and Hodge integrals in the tautological ring, with an appendix by Don Zagier, Michigan Math. J. (Fulton volume) 48 (2000), 215-252.

[10] _ Hodge integrals and Gromov-Witten theory, Invent. Math. 139 (2000) 173-199.

[11] E. Getzler, R. Pandharipande, Virasoro constraints and the Chern classes of the Hodge bundle, Nuclear Phys. B 530 (1998), no. 3, 701-714.

[12] E. Getzler, A. Okounkov and R. Pandharipande, Multipoint series of Gromov-Witten invariants of $\mathbb{C} P^{1}$, Lett. Math. Phys., 2002, 62(2), 159-170.

[13] A. Givental, Gromov-Witten invariants and quantization of quadratic Hamiltonians, Mosc. Math. J. 1 (2001), no. 4, 551C568, 645.

[14] I.P. Goulden, D.M. Jackson and R. Vakil, The moduli space of curves, double Hurwitz numbers and Faber's intersection number conjecture, math.AG/0611659.

[15] _ A short proof of the $\lambda_{g}$-conjecture without Gromov-Witten theory: Hurwitz theory and the moduli of curves, preprint, math.AG/0604297.

[16] R. Kaufmann, Yu. Manin, and D. Zagier, Higher Weil-Petersson volumes of moduli spaces of stable n-pointed curves, Comm. Math. Phys. 181 (1996), 763-787.

[17] M. Kontsevich, Intersection theory on the moduli space of curves and the matrix Airy function. Comm. Math. Phys. 147 (1992), no. 1, 1-23.

[18] K. Liu and $\mathrm{H} . \mathrm{Xu}$, Intersection numbers on $\overline{\mathcal{M}}_{g, n}$ and automorphisms of stable curves, math.AG/0608209.

[19] - The n-point functions for intersection numbers on moduli spaces of curves, math.AG/0701319.

[20] Looijenga, E., On the tautological ring of $\mathcal{M}_{g}$, Invent. Math., 121, (1995) 411C419.

[21] D. Mumford, Towards an enumerative geometry of the moduli space of curves, in Arithmetic and Geometry (M. Artin and J. Tate, eds.), Part II, Birkhäuser, 1983, 271-328.

[22] A. Okounkov, Generating functions for intersection numbers on moduli spaces of curves, Internat. Math. Res. Notices, (2002), 933-957.

[23] R. Vakil, The moduli space of curves and Gromov-Witten theory, preprint, math.AG/0602347.

[24] E. Witten, Two-dimensional gravity and intersection theory on moduli space, Surveys in Differential Geometry, vol.1, (1991) 243-310.

[25] D. Zagier, The three-point function for $\overline{\mathcal{M}}_{g}$, unpublished.

Center of Mathematical Sciences, Zhejiang Univ., Hangzhou, Zhejiang 310027, China; Dept. of Math., Univ. of California at Los Angeles, los Angeles, CA 90095-1555, USA

E-mail address: liu@math.ucla.edu, liu@cms.zju.edu.cn

Center of Math. Sciences, Zhejiang University, Hangzhou, Zhejiang 310027, China

E-mail address: haoxu@cms.zju.edu.cn 